\title{
Evaluation of a Wearable Hybrid Textile Antenna
}

\author{
H.M.R Nurul ${ }^{* 1}$, P.J Soh ${ }^{* \# 2}$, G.A.E Vandenbosch ${ }^{\# 3}$, M.F Malek ${ }^{* 4}$, S.L Ooi ${ }^{* 5}$, I.Adam ${ }^{* 6}$ \\ *School of Computer and Communication Engineering, Universiti Malaysia Perlis (UniMAP) \\ Block A, Seberang Ramai School Complex, 20000 Kuala Perlis, Perlis, MALAYSIA \\ ${ }^{1,5}$ \{nwhosena, sooliam\}@gmail.com \\ ${ }^{2,4,6}\{$ pj soh, mfareq, ismahayati\}@unimap.edu.my \\ ${ }^{\#}$ ESAT-TELEMIC, Katholieke Universiteit Leuven \\ Kasteelpark Arenberg 10, 3001 Leuven, BELGIUM \\ ${ }^{2,3}\{$ pingjack.soh, guy.vandenbosch\}@esat.kuleuven.be
}

\begin{abstract}
Due to its popularity, antennas implemented on conductive textiles has been widely investigated. Prototypes of the fully textile antennas are also usually pre-investigated using conductive foil which is more homogeneous and provides better mechanical consistency. However, in today's practical application, various electronic devices are still implemented on low-cost FR4 boards, popular in the printed circuit board (PCB) technology. This work aims to evaluate the case of a FR4-textile hybridization scheme, and compare it with another two types of antennas, which are implemented either on a similar-sized copper foil prototype and a fully textile single layered rectangular patch structure. Through the work done, it is then possible to better estimate the shift in terms of S11, due to the dissimilar conductivity and fringing fields with different dielectric load. A measure of gain-efficiency reduction when placed in proximity to a human body model in simulation is also evaluated. As a result of this investigation, the copper foil antenna is established to be producing the least gain reduction, and slightest S11 detuning on human arm. On the other hand, hybrid and fully textile antennas behaved very similarly in terms of S11 and bandwidth, both in free space and on arm.
\end{abstract}

\section{INTRODUCTION}

Due to the rapid development and widespread usage of the IEEE 802.11 wireless protocol, promising wearable structures have been proposed. In order to meet the Body Area Network (BAN) antenna requirements - size reduction and flexibility, diverse proposed structures derived from a multitude of materials have been investigated. These includes microstrip rectangular patches and its derivatives described in [1-2], circular [3], dipoles [4], and various slotted structures [5-6]. In practice, integration can be done as simply as interconnecting the device to the antenna via cable or wirelessly.

While all of the mentioned structures employ fully textile and/or metallic/substrate printed circuit board (PCB), investigation into wearable textile antennas have yet to determine the effects of hybridization of the structure, i.e a metallic/PCB integrated onto a textile based structure. The proposed hybrid structure is of interest in this investigation due to the current commercial electronic devices limitation, which is still implemented using conventional FR4 substrates. It also enables an estimated cohesion of a textile, or partially textile antenna performance when integrated into miniature electronic devices worn on the human body. This work attempts to implement a simple rectangular structure in order to achieve the said objective, through the implementation of two antennas fabricated totally either using conventional copper foil or conductive textile. These results are then compared with a hybrid FR4-conductive textile structure which is joint manually on a transmission line.

A rectangular microstrip antenna has been widely known as a classical structure, popular for low-profile applications at frequencies above $100 \mathrm{MHz}$. A bottom-fed structure is defined by its patch width, $W$, length, $L$, and height, $h$, which conventionally acts as a resonant $\lambda / 2$ parallel-plate structure. Due to its wide understanding of the structure and its simplicity in fabrication, it is chosen as the basis of this investigation.

Conventional antenna design uses soldering iron to connect between antenna's metallic transmission line and the SMA connector. However, conductive textile will not be robust enough to withstand the heat from soldering iron, thus an alternative method using conductive epoxy was applied in this experiment. Due to the importance in producing closest similarity possible when applying solder connection and epoxy connections, a hybrid prototype is fabricated, in comparison to a copper foil prototype, in order to evaluate its effect as a line, and then as an antenna. Finally, a simple, fully textile structure was also fabricated and evaluated for operation in proximity to the body.

\section{ANTENNA DESIGN}

The experimental conductive textile antenna was designed for the $2.45 \mathrm{GHz}$ ISM (Industrial, Scientific and Medical) frequency. In total, three structures are investigated - first, an antenna made fully from copper foil (antenna A), another is made from a hybrid FR4/textile structure (antenna B) and finally the third is made fully out of Zelt conductive textile (antenna $\mathrm{C}$ ). A standard $50 \Omega$ SMA connector is used to feed in RF power for testing. Antenna $\mathrm{A}$ and $\mathrm{B}$ are placed on an empty, standard-sized (25 x $20 \mathrm{~mm})$ FR4 board with an existent ground plane at the bottom. These FR4 boards are 


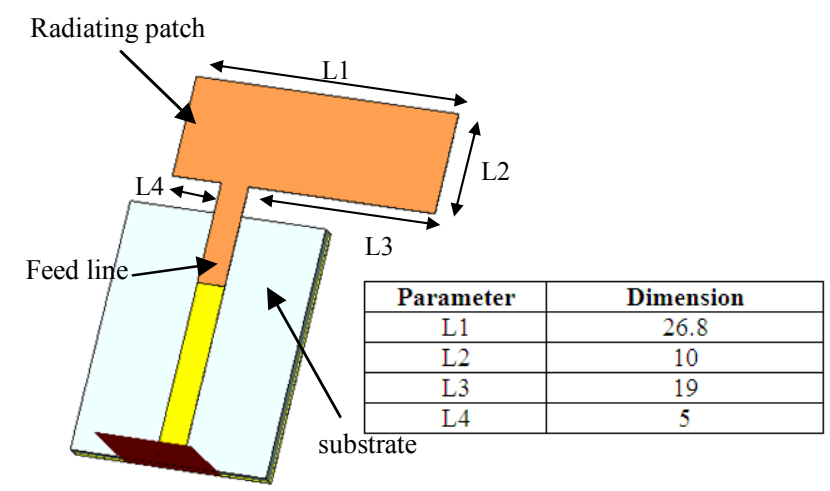

Fig. 1 Proposed rectangular patch antenna structure in this investigation

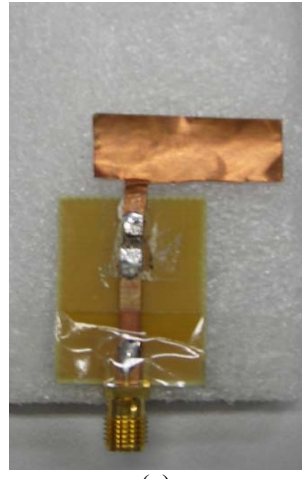

(a)

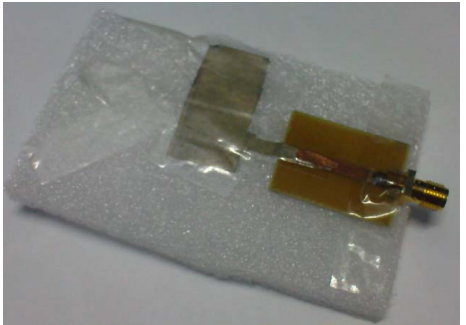

(b)

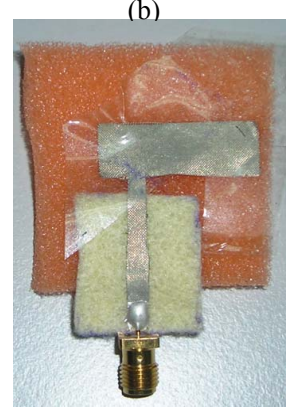

(c)
Fig. 2 Fabricated prototypes of the antennas (a) Copper foil rectangular patch antenna (Antenna A); (b) hybrid patch antenna (Antenna B) and (c) simple textile patch antenna (Antenna C)

$1.6 \mathrm{~mm}$ in thickness, with a relative permittivity, $\varepsilon_{r}$, of 4.7 . On the other hand, antenna $\mathrm{C}$, is designed on a $3 \mathrm{~mm}$ line etched on the top layer, exhibiting a $50 \Omega$ impedance. All extended areas of the patch has been measured practically using a supporting polystyrene foam, similar to air, with an estimated relative permittivity of $\varepsilon_{r}=1.02$. Design simulations of the antenna and microstrip line have been carried out using commercial electromagnetic solver, CST Microwave Studio.

The Zelt conductive fabric used in this design is composed of a copper coated, woven nylon taffeta fabric, having an estimated surface resistivity of $0.06 \Omega$ /square and thickness, $t$, of $0.0635 \mathrm{~mm}$. In simulation, the antenna is modelled as a lossy metal to reduce simulation time. Its conductivity, $\sigma$, is calculated based on the value of the surface resistivity, $R_{\mathrm{s}}$, and thickness of the textile material, $t$, as $\sigma=\left(1 /\left(R_{s} \times t\right)\right)$. This produced an estimated conductivity of $2.5 \times 10^{5} \mathrm{~S} / \mathrm{m}$, which was then defined in the electromagnetic solver.
In initiating the design of this antenna, the well-known basic rectangular patch design equation using the transmission line model as given in [7] was utilized. For the FR4 used in this work, it yielded an effective relative permittivity, $\varepsilon_{\text {reff }}$ of 5.27 and an estimated $L 1$ of $26.67 \mathrm{~mm}$, which is extremely close to the optimized value of $26.8 \mathrm{~mm}$. The antenna's $50 \Omega$ point at the radiating edge is estimated to be located at about three quarter of the patch's width $(0.75 \times 21)$, which yielded a calculated value of $6.67 \mathrm{~mm}$ from the edge. It can be seen that due to the $3 \mathrm{~mm}$-wide feedline, the distance $L 4$ is reduced to 5 $\mathrm{mm}$.

On the other hand, antenna $\mathrm{C}$ designed using fleece fabric as the substrate was assumed to have a relative permittivity of $\varepsilon_{r}=1.26$. This resulted in an $\varepsilon_{\text {reff }}$ of 1.3. To ensure a fair comparison with antennas $\mathrm{A}$ and $\mathrm{B}$, a similarly dimensioned patch antenna was fabricated using Zelt conductive fabric.

\section{RESULTS AND DISCUSSION}

\section{A. Sensitivity Analysis}

Due to the fabrication methods of the antennas which utilized simple manual cutting tools, it is important to investigate the effect of dimensional variations due to cutting inaccuracies. As can be observed from Figures 3 and 4, width and length of the patch ( $L 1$ and $L 2)$ and width and length of the substrate $(F R 4 W$ and $F R 4 L)$ are critical parameters in changing the reflection coefficient $\left(S_{11}\right)$. Each study involved the changing of $1 \mathrm{~mm}$ in length. As it is in classical theories, the elongation of $L 1$ and $L 2$ indicate the increment in wavelengths and produced lower resonance. $L 1$ was lowering the frequency by about $20 \mathrm{MHz}$ with each milimeter increment, while this frequency down-shift rate was doubled for $L 2$, about $40 \mathrm{MHz}$ per $1 \mathrm{~mm}$ increment.

On the other hand, size increment in substrate's width $(F R 4 W)$ and length (FR4L) moved the resonance higher. $F R 4 W$ changed the resonance by about 400 to $500 \mathrm{MHz}$ per milimeter of length increase, while $F R 4 L$ increased the resonance by about $950 \mathrm{MHz} / \mathrm{mm}$. Finally, the effect of the feeding position, $L 4$, was investigated by sweeping its length from $5 \mathrm{~mm}$ to $18 \mathrm{~mm}$. It was noticed that the relative distance from the center of the patch is determining antenna impedance. This explains the reason that when the feeding position is moved from left to right, a pair of identical response exists for a similar distance from center of the patch.

\section{B. S11 and Antenna Bandwidth}

Since similar dimensions are implemented in all three designs, difference of conductive material, substrate and feeding transitions is expected to affect the $S_{11}$ response. This is proven when the third antenna made out of conductive textile (Antenna C) was seen to be resonating at a slightly higher frequency of about $2.71 \mathrm{GHz}$, whereas Antenna A and $\mathrm{B}$ is resonating at the desired frequency of about $2.45 \mathrm{GHz}$. During measurement, all antennas were observed be produce smaller bandwidth and upwards frequency shift in comparison to respective simulated result. Antenna $\mathrm{A}$ is the worst affected 
TABLE I

Effect of Parameters' change on Resonant Frequency (fres)

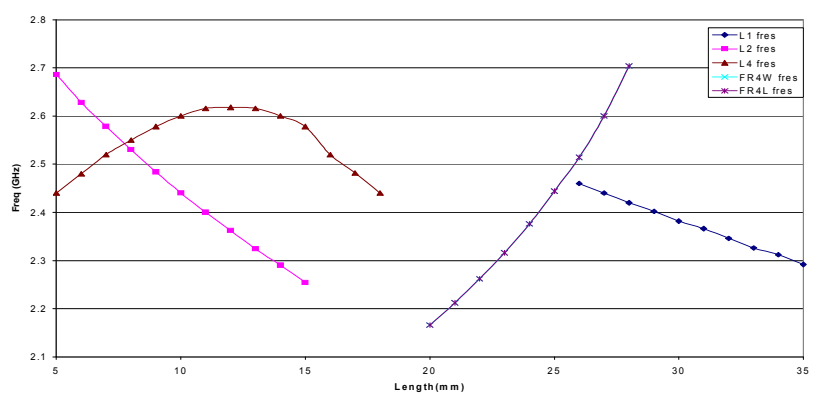

Fig. 3 Effect of various parameter changes on the resonant frequency

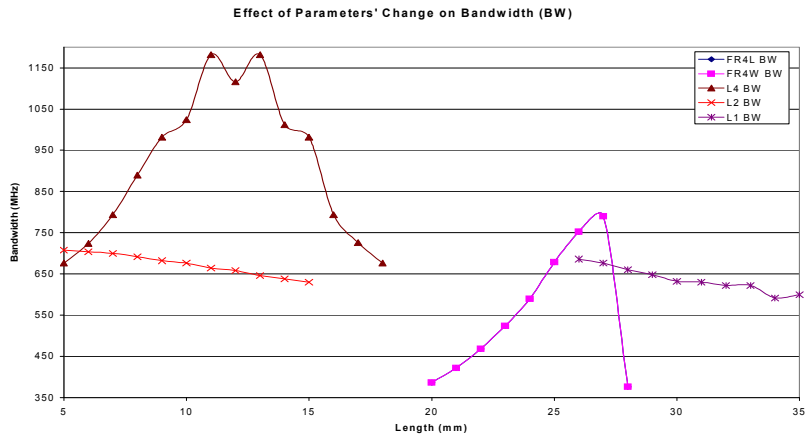

Fig. 4 Effect of various parameter changes on the bandwidth

in terms of $S_{11}$; producing a measured value of $2.64 \mathrm{GHz}$, while its bandwidth also shrunk by $180 \mathrm{MHz}$ in comparison to the simulated value of $700 \mathrm{MHz}$. Antenna $\mathrm{B}$ and $\mathrm{C}$ did not produce a lot of $S_{11}$ variation, but its bandwidth was lesser by about half of its simulated value. The drastic change in bandwidth owes to the fact that the structures were not modelled together with the contribution of the supporting foam, which is naturally (and slightly) affecting the antenna's impedance through coupling. This is especially true when no ground plane is covering the radiating structure at the bottom end. The nature of microstrip feed lines, which are prone to surface wave losses might also contributed to the deviation. From the investigation, it was also observed that antennas B and $\mathrm{C}$ produced very similar $S_{11}$ and bandwidth change when measured. The hybrid antenna B produced only $30 \mathrm{MHz}$ of difference against measurement, while antenna $\mathrm{C}$ produced about $50 \mathrm{MHz}$ when evaluated in similar perspective. This proves that even though the antennas were radiating at different frequencies, the modelling technique used was accurate enough to predict antenna performance in terms of $S_{11}$. Summary of the results are given in Table 1 .

\section{Performance in Proximity of Human Body}

The three prototypes antennas are further analysed performing in proximity of the human body. This was done both through simulations (a Hugo human model in CST) and measurements. The antennas are intended for placement on the arm of a user, with a spacing of about $8 \mathrm{~mm}$ from the skin; in order to account for maximum amount of clothing worn. Half of the human body was added into the model as to create
Simulated AND MEASURED $S_{1 l}$ FOR ALL DESIGNS

\begin{tabular}{|l|c|c|c|c|}
\hline Antenna & $\begin{array}{c}\text { Res } \\
\text { Freq } \\
\text { (GHz) }\end{array}$ & $\begin{array}{c}\text { Res Diff } \\
\text { (MHz) }\end{array}$ & $\begin{array}{c}\text { Bandwidth } \\
\text { (MHz) }\end{array}$ & $\begin{array}{c}\text { Bandwidth } \\
\text { Diff (MHz) }\end{array}$ \\
\cline { 1 - 2 } A (Sim) & 2.45 & \multirow{2}{*}{190} & $2.17-2.87$ & 700 \\
\cline { 1 - 3 } A (Meas) & 2.64 & & $2.50-3.02$ & 520 \\
\hline B (Sim) & 2.71 & \multirow{2}{*}{50} & $2.34-3.73$ & 1390 \\
\cline { 1 - 2 } B (Meas) & 2.74 & & $2.34-3.05$ & 710 \\
\hline C (Sim) & 2.45 & \multirow{2}{*}{30} & $2.21-2.81$ & 600 \\
\cline { 1 - 2 } \cline { 5 - 6 } C (Meas) & 2.50 & & $2.38-2.78$ & 400 \\
\hline
\end{tabular}

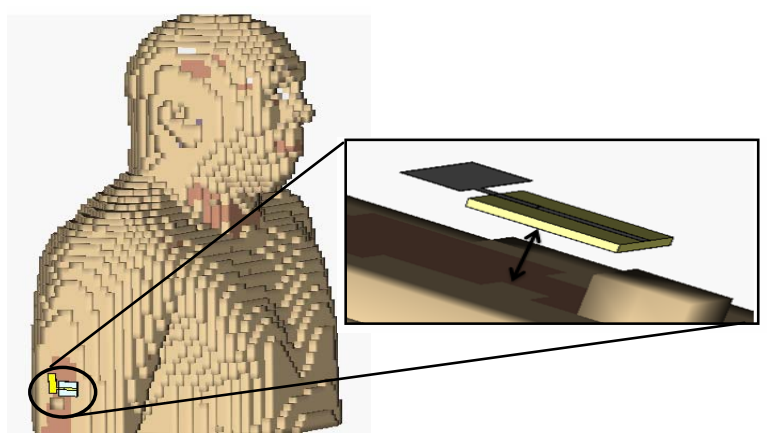

Fig. 5 Antenna simulation setup on human arm

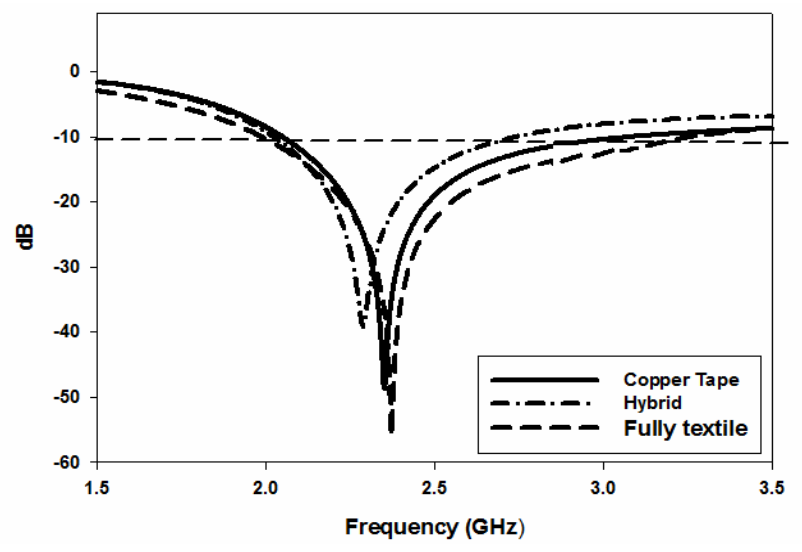

Fig. 6 Simulated S11 for all 3 antenna designs on human arm

a more comprehensive investigation, due to the antenna's proneness towards environmental coupling. Simulation setup is as shown in Fig 5, while their simulated and measured $S_{11}$ results are charted in Fig 6 and 7.

The observation showed excellent agreement among the three designs simulated using the Hugo body model in CST. For antenna $\mathrm{B}$, there is even noticeable improvement on the resonance and $S_{11}$ depth. However, when measured, only antenna A showed resonance consistency against its simulation, whereas antennas $\mathrm{B}$ and $\mathrm{C}$ indicated serious frequency up-shifting. All three antennas' radiation patterns are also similar when simulated in free space, in Fig 8. From Fig 9, it can be seen that the E-plane has been degraded by up to $10 \mathrm{~dB}$ for antennas $\mathrm{B}$ and $\mathrm{C}$, which are non- metallic, especially for radiation at the fourth quadrant. In general, 


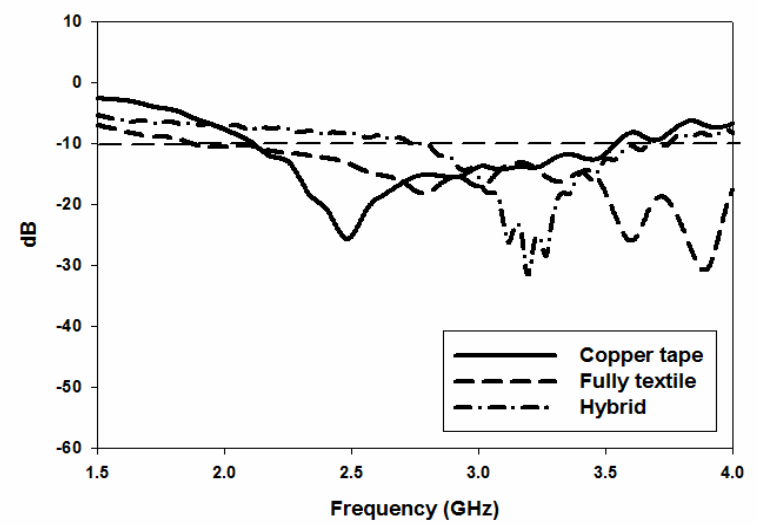

Fig. 7 Measured S11 for all 3 antenna designs on human arm

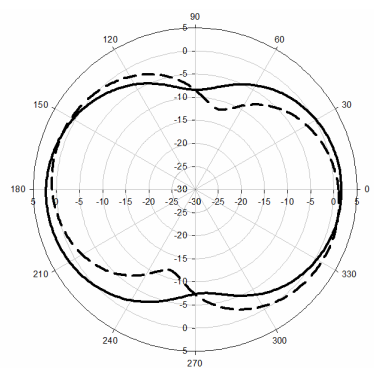

(a)

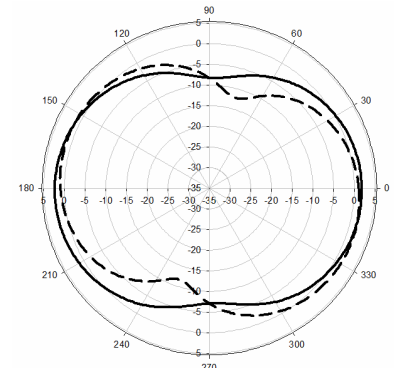

(b)

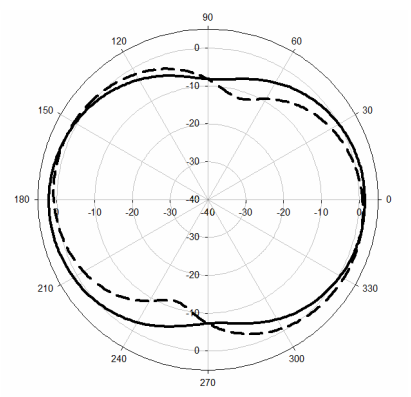

(c)

Fig. 8 Free space radiation pattern for (a) antenna A; (b) antenna B and (c) antenna C

TABLE III

GAIN AND EFFICIENCY FOR ALL DESIGNS

\begin{tabular}{|c|c|c|c|c|}
\hline Location & & Antenna A & Antenna B & Antenna C \\
\hline \multirow{2}{*}{$\begin{array}{c}\text { Free } \\
\text { space }\end{array}$} & $\begin{array}{c}\text { Gain } \\
(\mathrm{dB})\end{array}$ & 2.144 & 2.324 & 2.135 \\
\cline { 2 - 5 } & Eff $(\%)$ & 89.09 & 98.46 & 89.49 \\
\hline \multirow{2}{*}{ On body } & $\begin{array}{c}\text { Gain } \\
(\mathrm{dB})\end{array}$ & 0.8575 & 0.3650 & 0.3356 \\
\cline { 2 - 5 } & Eff $(\%)$ & 42.56 & 36.17 & 37.11 \\
\hline
\end{tabular}

antennas $\mathrm{B}$ and $\mathrm{C}$ changed similarly in resonance and bandwidth, while all three antennas' suffered loss in efficiency/gain, and direction change in radiation pattern when placed in close proximity to the arm. Efficiency was highly degraded for non metallic antennas $\mathrm{B}$ and $\mathrm{C}$ when placed on arm, decreasing from a value of about $90 \%$ to only $37 \%$, at best.

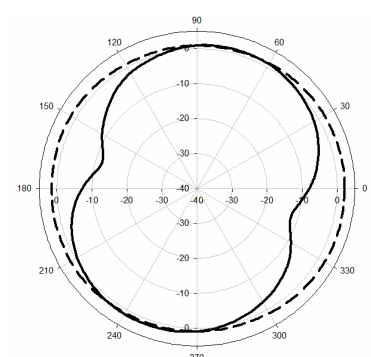

(a)

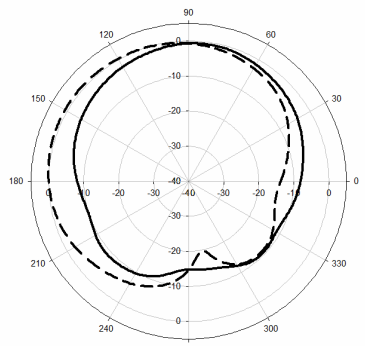

(b)

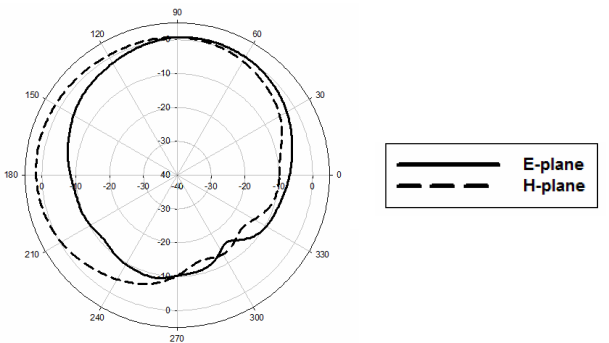

(c)

Fig. 9 On-body radiation pattern for (a) antenna A; (b) antenna B and (c) antenna $\mathrm{C}$

\section{CONCLUSIONS}

Three antenna prototypes with similar dimensions and varying material builds were investigated in this work in order to determine the validity of PCB-to-textile transitions. Hybrid antenna $\mathrm{B}$ is performing very similarly to fully textile antenna $\mathrm{C}$ in free space-both antennas are able to be designed with least variation from measurement values. On the other hand, metallic antenna A was the least affected when tested on human arm. The efficiency for a non-metallic antenna B and $\mathrm{C}$ was also found to be about half of its free space value, degrading by about $2 \mathrm{~dB}$ in gain and changing their radiation direction.

\section{REFERENCES}

[1] C. Hertleer, H. Rogier, L. Vallozzi, and L. Van Langenhove, "A Textile Antenna for Off-Body Communication Integrated Into Protective Clothing for Firefighters," Antennas and Propagation, IEEE Transactions on, vol. 57, pp. 919-925, 2009.

[2] C. Hertleer, A. Tronquo, H. Rogier, L. Vallozzi, and L. Van Langenhove, "Aperture-Coupled Patch Antenna for Integration Into Wearable Textile Systems," Antennas and Wireless Propagation Letters, IEEE, vol. 6, pp. 392-395, 2007.

[3] S. Sankaralingam and B. Gupta, "A Circular Disk Microstrip WLAN Antenna for Wearable Applications," in India Conference (INDICON), 2009 Annual IEEE, 2009, pp. 1-4.

[4] P. Salonen, M. Keskilammi, J. Rantanen, and L. Sydanheimo, "A novel Bluetooth antenna on flexible substrate for smart clothing," in Systems, Man, and Cybernetics, 2001 IEEE International Conference on, 2001, pp. $789-794$ vol.2.

[5] P. Salonen and M. Keskilammi, "SoftWear Antenna," in Military Communications Conference, 2008. MILCOM 2008. IEEE, 2008, pp. 1-

[6] P. Kabacik, R. Hossa, and A. Byndas, "Foldeable wideband small antenna element," in Antennas and Propagation Society International Symposium, 2007 IEEE, 2007, pp. 2598-2601.

[7] C. A. Balanis, Antenna theory: Wiley New York, 1997. 